\title{
Detection of Normal Aging Effects on Human Brain Metabolite Concentrations and Microstructure with Whole-Brain MR Spectroscopic Imaging and Quantitative MR Imaging
}

\author{
V.V. Eylers, (D.A. Maudsley, (DP. Bronzlik, P.R. Dellani, H. Lanfermann, and X.-Q. Ding
}

\begin{abstract}
BACKGROUND AND PURPOSE: Knowledge of age-related physiological changes in the human brain is a prerequisite to identify neurodegenerative diseases. Therefore, in this study whole-brain ${ }^{~} \mathrm{H}-\mathrm{MRS}$ was used in combination with quantitative MR imaging to study the effects of normal aging on healthy human brain metabolites and microstructure.
\end{abstract}

MATERIALS AND METHODS: Sixty healthy volunteers, 21-70 years of age, were studied. Brain maps of the metabolites NAA, creatine and phosphocreatine, and Cho and the tissue irreversible and reversible transverse relaxation times $\mathrm{T} 2$ and $\mathrm{T} 2$ ' were derived from the datasets. The relative metabolite concentrations and the values of relaxation times were measured with ROls placed within the frontal and parietal WM, centrum semiovale, splenium of the corpus callosum, hand motor area, occipital GM, putamen, thalamus, pons ventral/dorsal, and cerebellar white matter and posterior lobe. Linear regression analysis and Pearson correlation tests were used to analyze the data.

RESULTS: Aging resulted in decreased NAA concentrations in the occipital GM, putamen, splenium of the corpus callosum, and pons ventral and decreased creatine and phosphocreatine concentrations in the pons dorsal and putamen. Cho concentrations did not change significantly in selected brain regions. T2 increased in the cerebellar white matter and decreased in the splenium of the corpus callosum with aging, while the $\mathrm{T2}^{\prime}$ decreased in the occipital GM, hand motor area, and putamen, and increased in the splenium of the corpus callosum. Correlations were found between NAA concentrations and T2' in the occipital GM and putamen and between creatine and phosphocreatine concentrations and $\mathrm{T}^{\prime}$ ' in the putamen.

CONCLUSIONS: The effects of normal aging on brain metabolites and microstructure are region-dependent. Correlations between both processes are evident in the gray matter. The obtained data could be used as references for future studies on patients.

ABBREVIATIONS: $\mathrm{BSd}=$ pons dorsal; $\mathrm{BSv}=$ pons ventral; $\mathrm{HK}=$ hand motor area; $\mathrm{SCC}=$ splenium of the corpus callosum; $\mathrm{tCr}=\mathrm{creatine}$ and phosphocreatine

A s a prerequisite to identify neurodegenerative diseases, the knowledge of age-related physiologic changes in the human brain becomes more important in a society with increasing life expectancy. ${ }^{1} \mathrm{H}-\mathrm{MR}$ spectroscopy and quantitative MR imaging of brain tissue are both useful tools to study these changes. ${ }^{1} \mathrm{H}-\mathrm{MR}$ spectroscopy enables detection of brain metabolites, which, for example, reflect neuronal attenuation and

Received March 16, 2015; accepted after revision July 26.

From the Institute of Diagnostic and Interventional Neuroradiology (V.V.E., P.B., P.R.D., H.L., X.-Q.D.), Hannover Medical School, Hannover, Germany; and Department of Radiology (A.A.M.), University of Miami School of Medicine, Miami,

Florida.

This work was partially supported by Deutsche Forschungsgemeinschaft and by the National Institutes of Health grants R01 EB016064 and R01 EB008222 (A.A.M.)

Please address correspondence to Xiao-Qi Ding, MD, Institute of Diagnostic and Interventional Neuroradiology, Hannover Medical School, Carl-Neuberg-Str One, 30625 Hannover, Germany; e-mail: ding.xiaoqi@mh-hannover.de

-- Indicates open access to non-subscribers at www.ajnr.org

http://dx.doi.org/10.3174/ajnr.A4557 integrity (NAA), relate to the turnover of cell membranes (Cho), or serve as a marker of energy metabolism (creatine and phosphocreatine $[\mathrm{tCr}]$ ). Thus, ${ }^{1} \mathrm{H}-\mathrm{MR}$ spectroscopy has often been applied to study age-associated neurometabolism ${ }^{1}$ or pathology in the human brain. ${ }^{2}$ Due to limitations in the spatial coverage of standard acquisition techniques, most of the ${ }^{1} \mathrm{H}$-MR spectroscopy studies have been performed by using just 1 or a few small brain regions. ${ }^{1}$ In recent years, a wholebrain ${ }^{1} \mathrm{H}$-MRS technique with high spatial resolution has been established and has been used to study metabolic aging effects by deriving mean metabolite concentrations within each brain lobe. ${ }^{3}$ However, the evaluation of aging effects in multiple, more differentiated anatomic structures in human brain remains unreported, to our knowledge.

Quantitative MR imaging methods can be used to quantify relaxation processes of brain tissue, such as transverse relaxation due to spin-spin interactions characterized by the irreversible relaxation time (T2), local magnetic field inhomoge- 
neity characterized by the reversible relaxation time $\left(\mathrm{T} 2^{\prime}\right)$, or both mechanisms together characterized by an effective relaxation time $\left(\mathrm{T} 2^{*}\right)$, with the relation of $1 / \mathrm{T} 2^{*}=1 / \mathrm{T} 2+1 / \mathrm{T} 2^{\prime}$. The variations of transverse relaxation times reflect influences from the molecular environment within the tissue and provide a measure of microstructural changes in the maturing or aging brain $^{4-6}$ and brain diseases. ${ }^{7,8}$

In this prospective study, $\mathrm{NAA}, \mathrm{tCr}$, and Cho concentrations and T2 and T2' values were measured simultaneously in selected brain structures by using whole-brain ${ }^{1} \mathrm{H}-\mathrm{MRS}$ and quantitative MR imaging with the aim of detecting physiologic changes of the metabolism and microstructure of the human brain related to normal aging and possible correlations among metabolic and microstructural changes, which may provide insight into the effects of aging.

\section{MATERIALS AND METHODS Subjects}

Sixty-eight healthy volunteers were recruited from the local population. Based on a self-reporting interview, all subjects had no history of brain trauma, neurologic disorder, or other systemic diseases. Each subject received 2 screening tests (DemTect and the Beck Depression Inventory II) to exclude cognitive or psychiatric impairments. ${ }^{9,10}$ Sixty subjects $21-70$ years of age (mean age, $44 \pm 14$ years; 6 men and 6 women in each age group-ie, 21-30 years, group $1 ; 31-40$ years, group $2 ; 41-50$ years, group 3 ; 51-60 years, group 4; and 61-70 years, group 5) were included in the study. Eight subjects were excluded after the screening tests or due to incomplete MR imaging examination findings. The study was approved by local institution review board, and written consent was obtained from all subjects before the examinations.

\section{MR Imaging Examinations}

All MR imaging examinations were performed at 3T (Verio; Siemens, Erlangen, Germany). A 12-channel phased array head coil was used. The MR imaging protocol included a T2-weighted turbo spin-echo sequence with 3 echoes (triple TE) (TR/TE, 6640/ $8.7 / 70 / 131 \mathrm{~ms} ; 150^{\circ}$ flip angle; 3 -mm section thickness; $256 \times 208$ matrix; $256 \times 208 \mathrm{~mm}$ FOV; acceleration factor, 2), a T2*weighted gradient-echo sequence with triple TEs (TR/TE, 1410/ $6.42 / 18.42 / 30.42 \mathrm{~ms} ; 20^{\circ}$ flip angle; $3-\mathrm{mm}$ section thickness; $256 \times 208$ matrix; $256 \times 208 \mathrm{~mm}$ FOV; acceleration factor, 2), and volumetric spin-echo-planar spectroscopic imaging (TR/TE, $1710 / 70 \mathrm{~ms}$ ) acquisition by using an FOV of $280 \times 280 \times 180$ $\mathrm{mm}^{3}$ and a slab of $135 \mathrm{~mm}$, with a resolution of approximately 1 mL. ${ }^{11}$ The spin-echo-planar spectroscopic imaging acquisition included a second dataset obtained without water suppression that provided a water reference signal with spatial parameters identical to those of the metabolite MR spectroscopy. The water reference MR spectroscopic imaging was used for several processing functions, including measurement and correction of the resonance frequency offset at each voxel location, correction of line shape distortions, and providing an internal signal reference for the normalization of metabolite concentrations. ${ }^{12}$ In addition, a T1-weighted 3D MPRAGE image was obtained at 1-mm isotropic resolution for anatomic reference. The spin-echo-planar spectroscopic imaging, MPRAGE, TSE, and gradient-echo scans were obtained with the same angulation so that the same anatomic structures could be identified. The total scan time was approximately 36 minutes.

\section{Data Processing}

T2- and T1-weighted images were inspected by 2 experienced neuroradiologists to exclude subjects with morphologic abnormalities.

Whole-Brain 'H-MRS and Metabolite Maps. Reconstructions of the metabolite and water reference images were made as described by Maudsley et $\mathrm{al}^{3}{ }^{3}$ on which the spin-echo-planar spectroscopic imaging data were analyzed by using the Metabolic Imaging and Data Analysis software to determine volumetric maps of the metabolites NAA, tCr, and Cho, with metabolite signal-intensity normalization by using tissue water as an internal reference and the corresponding maps of spectral line width. All resultant maps were spatially transformed and interpolated to a standard spatial reference at 2-mm isotropic resolution.

Maps of Relaxation Times. T2 and $\mathrm{T}^{\star}$ maps were obtained on the fly on the MR imaging system with an extended image reconstruction provided by the manufacturer, by using monoexponential fitting to the signal-intensity decay curves of the triple TE data acquired with TSE and gradient-echo sequences, respectively. T2' values were calculated according to the relationship $1 / \mathrm{T} 2^{\prime}=$ $1 / \mathrm{T} 2 *-1 / \mathrm{T} 2$.

ROI Analysis. Local metabolite concentrations NAA, tCr, and Cho, as a ratio to tissue water, together with the spectral line width and tissue relaxation times $\mathrm{T} 2$ and $\mathrm{T} 2{ }^{\prime}$, were determined by using mean values over ROIs at 13 locations within each hemisphere: the frontal and parietal WM, centrum semiovale, splenium of the corpus callosum (SCC), hand motor cortex (HK), occipital GM, putamen, pallidum, thalamus, pons ventral (BSv), pons dorsal (BSd) at the level of the upper pons, and cerebellar white matter at the axial level of the midcerebellar peduncle and cerebellar posterior lobe. Metabolite values obtained from spectra with a line width of $>12 \mathrm{~Hz}$ or with a signal-to-noise ratio of $<5$ were not sampled. Paired $t$ tests showed that there were significant differences $(P<.05)$ of up to $10 \%$ between the bihemispheric values at several ROIs. However, considering the limitations of the sample size related to the handedness ( 54 for right-handed versus 6 for left-handed), the left and right values for each ROI were averaged for further analysis. Due to local magnetic field distortion caused by neighboring structures containing bone and air, ROIs at the genu of the corpus callosum and caudate nucleus were not considered. All ROIs were carefully drawn within a single section on T1-weighted images (for metabolite measurements) and T2weighted images (for quantitative MR imaging measurements) as a circle with an area of $29 \pm 4 \mathrm{~mm}^{2}$, which was manually located according to anatomic landmarks to exclude partial volume effects. ROI measurements on the relaxation time maps were made by using ImageJ software (National Institutes of Health, Bethesda, Maryland), ${ }^{13}$ while those on the metabolites were done with the ROI tool in the Metabolic Imaging and Data Analysis software. No CSF correction was made considering that the ROIs were placed within brain tissue. 


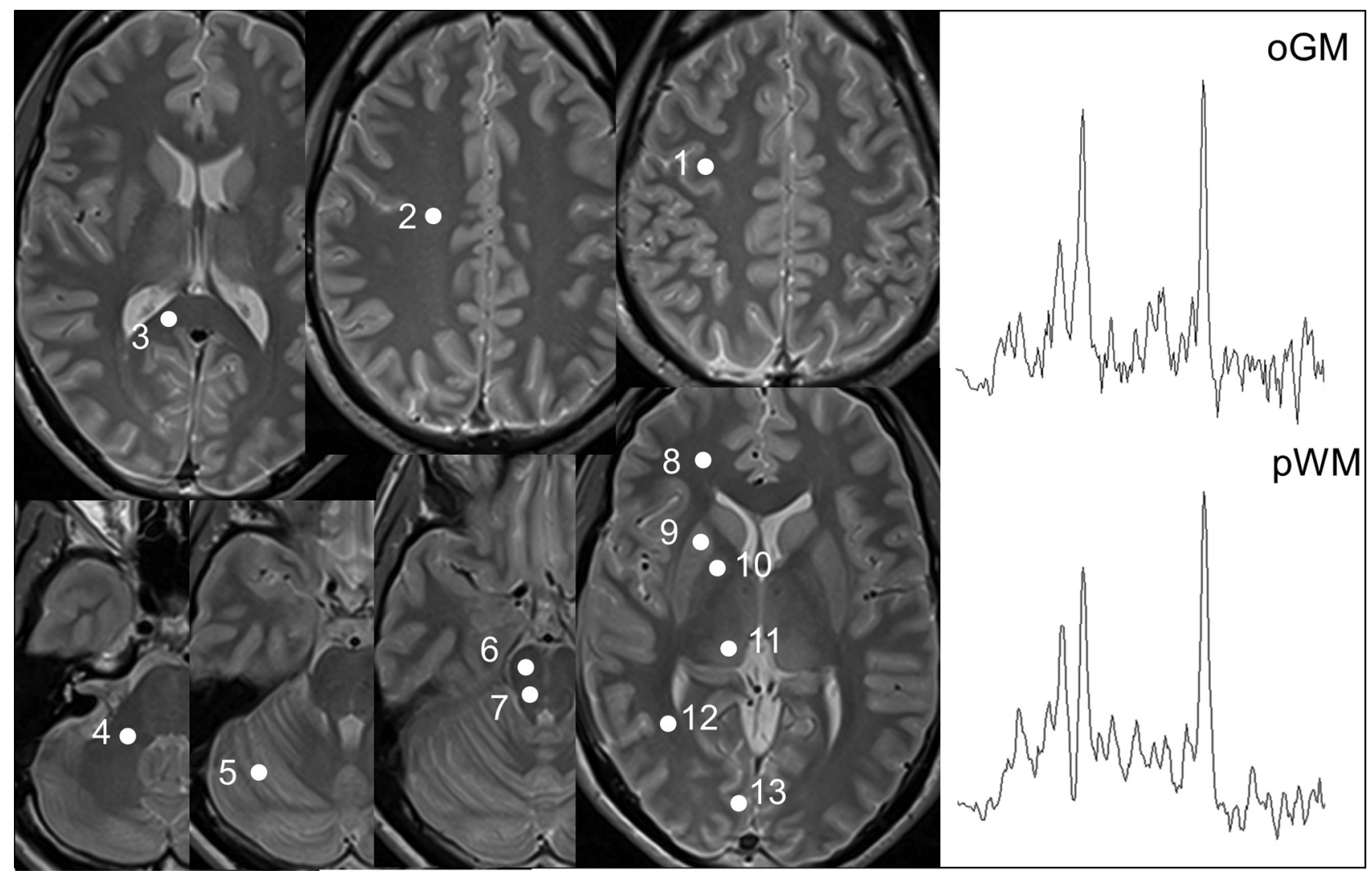

FIG 1. Locations of each selected ROI in the right brain hemisphere displayed as white filled circles on the T2-weighted images, respectively, and sample spectra from the parietal white matter and occipital gray matter of a 31-year-old volunteer. The numbering represents the ROIs in the hand motor cortex (1), centrum semiovale (2), splenium of the corpus callosum (3), cerebellar white matter (4) and posterior lobe (5), brain stem ventral (6) and dorsal (7), frontal white matter (8), putamen (9), pallidum (10), thalamus (11), parietal white matter (pWM, 12), and occipital gray matter (oGM, 13), respectively.

\section{Statistical Analysis}

For each ROI, the 2 -sided $t$ tests with Bonferroni correction (significance level, $\alpha=.05 / 3=.017$ ) were used to estimate sex differences of the measured local metabolite concentrations or transverse relaxation times. A linear regression analysis was used to estimate the age-dependence of the measured values, and the Pearson correlation test was used to test for possible correlations between changes of local metabolite concentrations and local transverse relaxation times (ie, to quantify the degree to which they are related). For linear regression analysis and for the Pearson correlation test, a low significance level of .01 was used to avoid accidental associations. Statistical analyses were performed with SPSS, Version 21 (IBM, Armonk, New York).

\section{RESULTS}

Locations of each selected ROI in the right-brain hemisphere are shown in Fig 1 as white filled circles on the T2-weighted images of a 31-year-old volunteer, where sample spectra in the parietal white matter and occipital gray matter are also shown. Metabolite maps of NAA, Cho, and $\mathrm{tCr}$ and maps of transverse relaxation times were obtained for all subjects for further ROI analyses.

The values of NAA, Cho, $\mathrm{tCr}$ concentrations, and $\mathrm{T} 2$ and $\mathrm{T} 2{ }^{\prime}$ were measured at each ROI on the corresponding maps of metabolites or relaxation times for all subjects. The group mean values of each age group obtained in corresponding ROIs are shown in Fig 2, where both metabolite concentrations and the transverse relaxation times show clear differences between the ROIs, indicating that the brain metabolite content or relaxation times are brain region-dependent.

Two-sided $t$ tests revealed no significant sex differences for most measures with the exceptions of NAA concentrations and $\mathrm{T} 2{ }^{\prime}$ in BSd, $\mathrm{tCr}$ concentrations in $\mathrm{HK}$, and $\mathrm{T} 2{ }^{\prime}$ in the cerebellar posterior lobe and centrum semiovale. Therefore, values for male and female subjects were combined for most ROIs, and those showing significant sex differences were analyzed separately for men and women. While the spectral line width at all ROIs did not reveal any significant correlation to age, linear regression analysis revealed significant $(P<.01)$ changes of metabolite concentrations and transverse relaxation times with age in most of the selected brain structures, as shown in the Table: NAA concentration was significantly decreased in the occipital GM, putamen, SCC, and BSv, with amounts varying from $-16 \%$ to $-26 \%$ within the observed 5 decades, and showed a trend of reduced values in the centrum semiovale $(R=-0.292, P=.024)$ and parietal WM $(R=-0.288, P=.026)$ and the thalamus $(R=-0.301, P=.019)$ (not shown). $\mathrm{tCr}$ concentration was reduced significantly in the putamen $(-17 \%)$ and dorsal brain stem $(-16 \%)$ and showed a tendency of increase in frontal WM $(R=0.27, P=.044)$ (not shown). Cho concentration in selected ROIs did not change significantly with age but had a small increase in frontal WM $(R=$ $0.261, P=.054)$. T2 decreased with age in the SCC $(-14 \%)$ and 


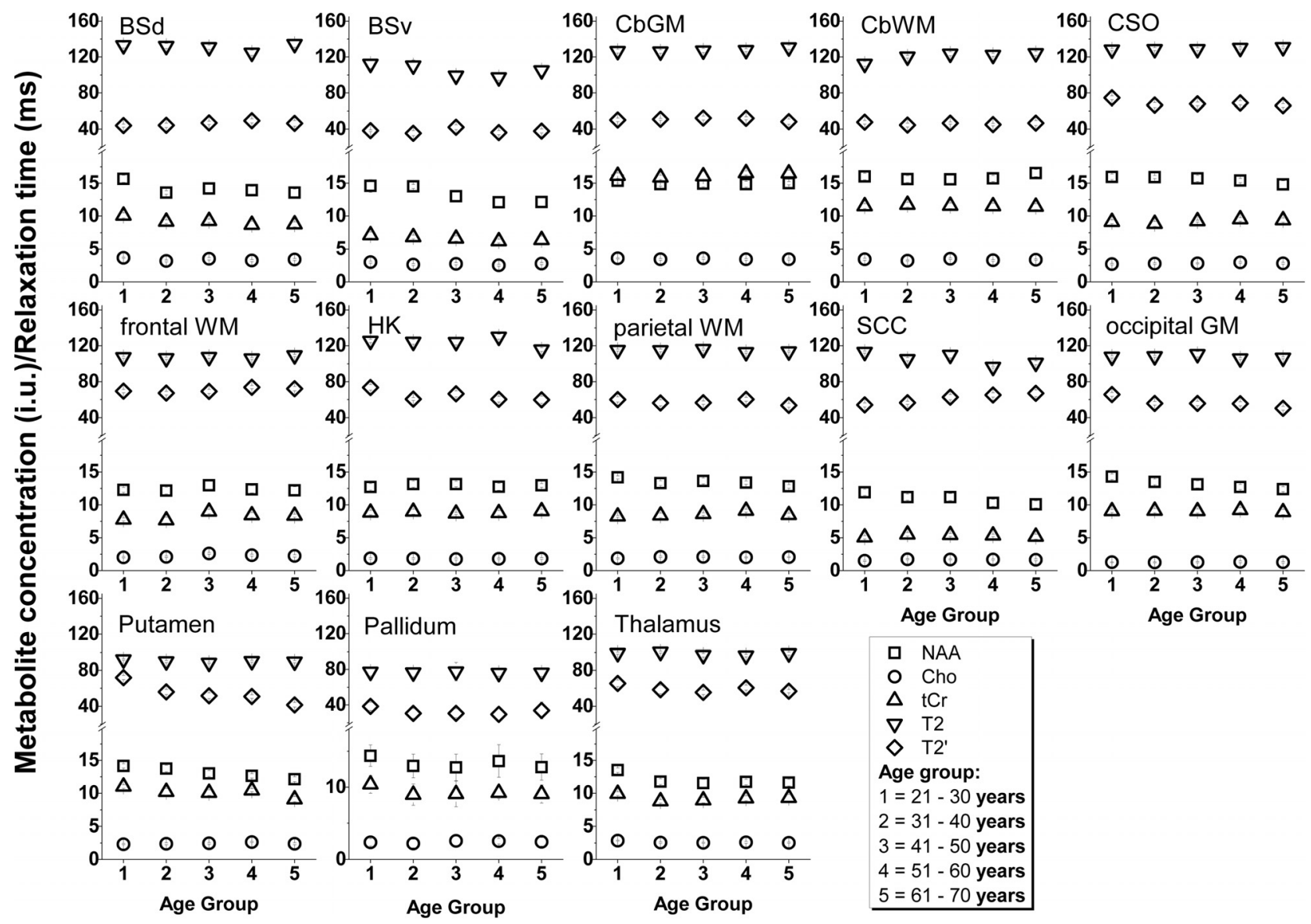

FIG 2. Group mean values of metabolite concentrations and tissue transverse relaxation times (y axis) with SDs for age group 1 (21-30 years), group 2 (31-40 years), group 3 (41-50 years), group 4 (51-60 years), and group 5 (61-70 years) are shown. The ROls are as described in Fig 1.

Significant associations $(P<.01)$ of the regional metabolite concentrations and transverse relaxation times with age, derived with linear regressions

\begin{tabular}{|c|c|c|c|c|c|c|c|c|c|}
\hline ROI & Parameter & Mean $^{a}$ & SD & $R$ & $P$ & No. ${ }^{b}$ & Intercept & Slope & Variation in $50 \mathrm{Yr}(\%)^{\mathrm{c}}$ \\
\hline \multirow[t]{2}{*}{ Occipital GM } & NAA & 13.2 & 1.6 & -0.40 & .002 & 59 & 15.16 & -0.04 & -16 \\
\hline & $\mathrm{T} 2^{\prime}$ & 56.7 & 9.4 & -0.45 & .000 & 60 & 69.67 & -0.30 & -23 \\
\hline \multirow[t]{3}{*}{ Putamen } & NAA & 13.2 & 1.5 & -0.51 & .000 & 60 & 15.54 & -0.05 & -19 \\
\hline & $\mathrm{tCr}$ & 10.2 & 1.4 & -0.39 & .002 & 60 & 11.89 & -0.04 & -17 \\
\hline & $\mathrm{T} 2^{\prime}$ & 55.1 & 13.1 & -0.68 & .000 & 60 & 83.44 & -0.66 & -47 \\
\hline Pallidum ${ }^{d}$ & $\mathrm{~T}^{\prime}$ & 32.3 & 6.4 & -0.34 & .005 & 40 & 41.53 & -0.24 & $-26^{\mathrm{e}}$ \\
\hline HK & $\mathrm{T}^{\prime}$ & 63.6 & 10.5 & -0.35 & .007 & 60 & 76.72 & -0.28 & -20 \\
\hline \multirow[t]{3}{*}{ SCC } & NAA & 10.9 & 1.9 & -0.36 & .005 & 60 & 13.01 & -0.05 & -19 \\
\hline & $\mathrm{T} 2$ & 105.1 & 9.3 & -0.47 & .000 & 60 & 118.50 & -0.31 & -14 \\
\hline & $\mathrm{T} 2^{\prime}$ & 57.5 & 7.4 & 0.40 & .002 & 60 & 45.14 & 0.36 & 35 \\
\hline CbWM & $\mathrm{T} 2$ & 120.5 & 8.3 & 0.45 & .000 & 60 & 108.93 & 0.26 & 12 \\
\hline BSv & NAA & 13.4 & 2.4 & -0.42 & .003 & 50 & 16.73 & -0.08 & -26 \\
\hline BSd & $\mathrm{tCr}$ & 9.2 & 1.3 & -0.35 & .010 & 53 & 10.64 & -0.03 & -16 \\
\hline
\end{tabular}

Note:- $R$ indicates the Pearson correlation coefficient.

${ }^{a}$ In ratio to internal water for metabolites and in milliseconds for relaxation times.

${ }^{b}$ Values of several ROls were not sampled for all subjects $(n<60)$ due to artifacts.

${ }^{\mathrm{C}}$ In ratio to the values at 20 years of age.

d The values for subjects older than 60 years were not sampled for linear regression.

e Variation in 40 years. Significant linear relation of T2' to age in the pallidum was found for the ages between 20 and 60 years $(P<.01)$, but not for the whole age range (21-70 years, $P>.01)$.

increased in the cerebellar white matter (12\%), and T2' decreased in occipital GM $(-23 \%)$, putamen $(-47 \%)$, and hand motor cortex $(-20 \%)$ and increased in the SCC (35\%) with age.

In Fig 3, the values of metabolite concentrations (top) and tissue transverse relaxation times (bottom) measured at the ROIs of the occipital GM, putamen, and pallidum are drawn according to age, together with the corresponding results of linear regression analysis: The NAA and T2' in the occipital GM and the NAA, tCr, and $\mathrm{T}^{\prime}{ }^{\prime}$ in the putamen revealed linear reductions with age $(P<.01$, Table), while tCr, Cho, and T2 in the occipital GM and Cho and T2 in the putamen did not show any age dependence. The results measured in the pallidum showed a tendency of de- 


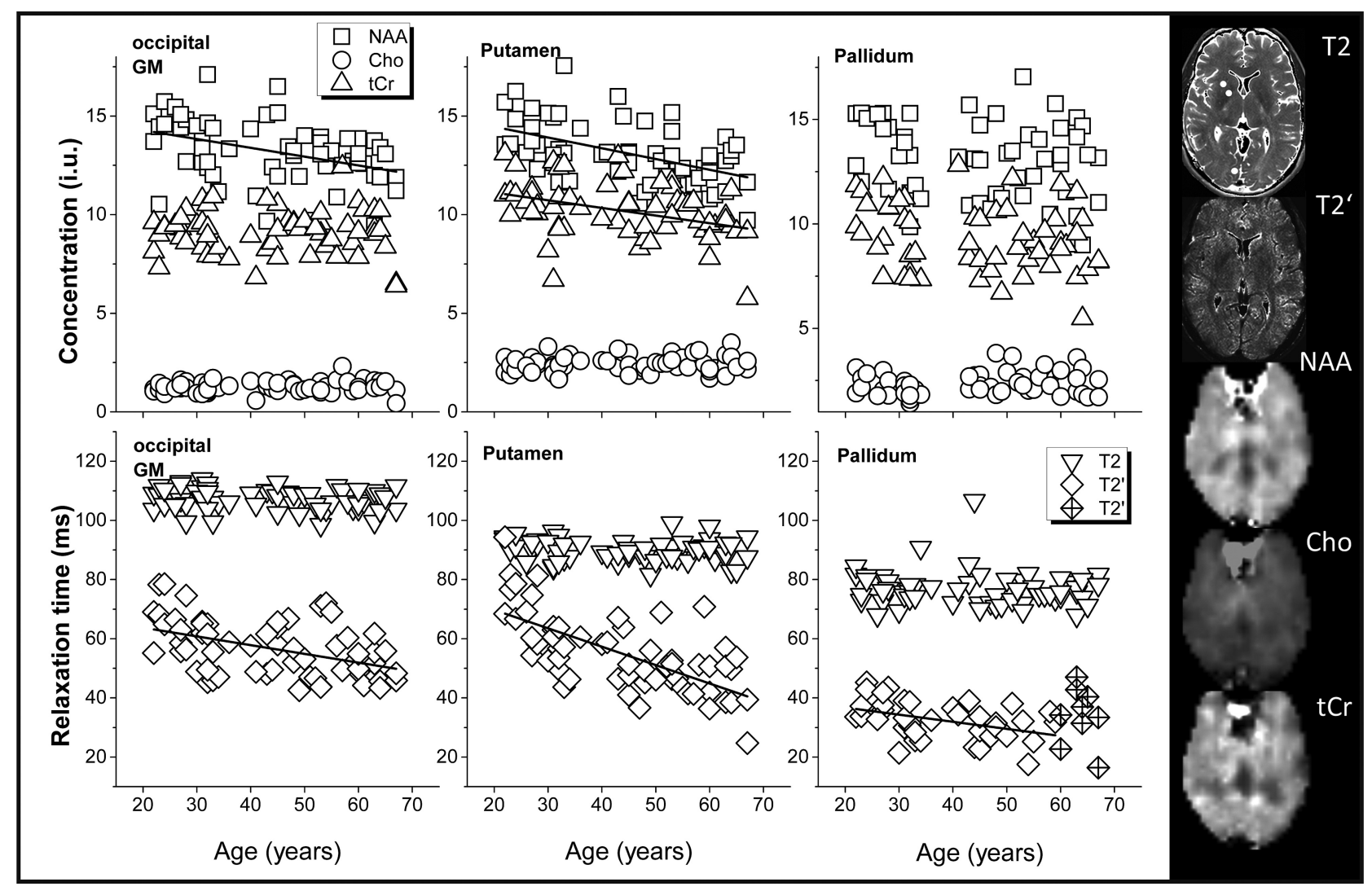

FIG 3. Values of the metabolite concentrations (top) and tissue transverse relaxation times (bottom) of all subjects measured at the ROls of occipital GM, putamen, and pallidum drawn according to the age, where NAA and T2' in the occipital GM and NAA, tCr, and T2' in the putamen reveal linear reductions with age ( $P<.01$ with the value of each slope being given in the Table), while tCr, Cho, and T2 in the occipital GM and Cho and $\mathrm{T} 2$ in putamen do not show an age dependence. No significant age correlations were found in the pallidum both for metabolites and relaxation times, but a significant linear reduction of $T 2^{\prime}$ with age was found up to 60 years of age $(P<.01$ with the value of each slope being given in the Table).

creased tCr with age $(R=-0.28, P=.04>.01)$ and no significant age correlation for NAA, Cho, and T2. T2' values in the pallidum were the lowest ones in comparison with all other ROIs and revealed a linear reduction with ages from 20 to 60 years $(-26 \%)$ $(P<.01$, Table $)$, though without further significant reduction for older than 60 years of age $(P=.73)$. The Pearson correlation tests showed significant positive correlations $(R=0.53, P<.01)$ between NAA concentrations and T2' in the occipital cortical gray matter, between NAA concentrations and T2' $(R=0.55, P<.01)$, and between $\mathrm{tCr}$ concentration and $\mathrm{T}^{\prime}(R=0.50, P<.01)$ in the putamen. Weak correlations between NAA concentrations and T2 $(R=0.30, P=.02)$ and between NAA concentrations and T2 ${ }^{\prime}(R=$ $-0.27, P=.03$ ) were found in the SCC.

\section{DISCUSSION}

Previous studies have examined associations of brain metabolite concentrations with age in selected brain structures with varying results, ${ }^{1}$ but few of these studies have examined a large number of brain anatomic structures. To our knowledge, no previous studies have examined possible associations with tissue relaxation times. The metabolite concentrations determined in this study agree in general with those reported by using single-voxel spectroscopy, with NAA being the largest component in the cerebrum and $\mathrm{tCr}$, the largest component in cerebellum, while the Cho concentration is the smallest in both regions, ${ }^{14,15}$ though in this study the measurements were obtained in multiple regions by using only a single MR spectroscopic acquisition. The T2 and T2' values found in this study are also comparable with those previously published, with $\mathrm{T} 2$ ' being shortest in the supratentorial deep gray matter, and $\mathrm{T} 2$, longest in the cerebellum. ${ }^{6,16}$

With combined whole-brain ${ }^{1} \mathrm{H}-\mathrm{MRSI}$ and quantitative MR imaging measurements, this study determined for the first time, simultaneously, the age-related metabolite concentrations and tissue transverse relaxation times at multiple supratentorial and infratentorial structures in the healthy human brain, which have the potential to provide a reference basis for future studies to identify pathologic alterations in patients. Additionally, with a significance level of .01, variable age dependences of the metabolite concentrations and the tissue relaxation times within the brain have been found. With increasing age, NAA changed the most among the 3 metabolites, with concentrations decreasing significantly in 4 ROIs (occipital GM, putamen, SCC, and BSv), while the $\mathrm{tCr}$ concentration decreased significantly in 2 ROIs (putamen, BSd). Cho concentration revealed only a tendency to increased values in the frontal white matter. Although the metabolite T2s were not directly measured, the observations of no significant correlations between age and spectral line width for all ROIs indicate that the changes in metabolite $\mathrm{T} 2 \mathrm{~s}$ with age are negligible. Thus, the possible influence on local metabolite concentrations 
caused by variations of metabolite T2s with age was not considered. For transverse relaxation times, the aging effect was most obvious for T2', which showed significant age-related changes in 4 ROIs, the occipital GM, putamen, hand motor cortex, and SCC, and some changes in the pallidum, while $\mathrm{T} 2$ significantly changed with age in only 2 ROIs (SCC and cerebellar white matter).

These observations indicate that NAA and $\mathrm{T} 2$ ' are sensitive to brain aging. Moreover, the observed age-related metabolic and microstructural changes were strongly brain region-dependent: The most significant changes occurred in the putamen (reducing NAA and tCr concentrations and T2') and the SCC (reducing NAA concentrations and T2, and increasing T2'), indicating that both structures are vulnerable to metabolic and microstructural aging. These observations are in line with previous reports that both the putamen and corpus callosum play important roles concerning age-related cognitive and motor impairment. ${ }^{17,18}$

Decreased brain NAA with age in several brain structures has also been reported previously by different authors. ${ }^{3,19,20}$ It has been suggested that decreased NAA concentrations reflect reduced neuronal attenuation or neuronal function. ${ }^{19,21}$ Both reasons could be responsible for our observations of age-related NAA concentration reductions. While the age-related reductions of NAA concentration are frequently reported, findings for Cho and tCr in relation to the age reported until now are more divergent. Raininko and Mattsson, ${ }^{22}$ in a single-voxel spectroscopy study at $1.5 \mathrm{~T}$, found age-related reduction of NAA concentration, but ageindependent Cho concentrations and $\mathrm{tCr}$ concentrations in cerebral supraventricular white matter. Charles et $\mathrm{al}^{20}$ found lower NAA, Cho, and tCr concentrations in the cortical and subcortical gray matter of older subjects but not in the white matter. In a single-voxel spectroscopy study at $1.5 \mathrm{~T}$ on 50 healthy subjects 20-70 years of age, Brooks et $\mathrm{al}^{19}$ found that the NAA decreased significantly with age in the interhemispheric tissue of the medial frontal lobe, with an overall decrease of $12 \%$ between the third and seventh decades, and the concentrations of $\mathrm{Cr}$ and Cho did not change significantly with age. Similar to our findings in the brain stem (decreased NAA concentrations at the BSv and decreased $\mathrm{tCr}$ concentrations at the BSd), an age-related decline of NAA and a trend toward decreased $\mathrm{tCr}$ concentrations in the upper brain stem were also observed by Moreno-Torres et $\mathrm{al}^{23}$ in a single-voxel spectroscopy study on 57 healthy subjects from 23 to 79 years of age. Therefore, more MR spectroscopy studies under standardized conditions will be necessary to obtain more precise and comparable values for multicenter studies to clarify these divergent findings.

It is interesting to compare our results with the lobar mean metabolite content derived by Maudsley et $\mathrm{al},{ }^{3}$ with the same spin-echo-planar spectroscopic imaging technique on a different sample of healthy volunteers. While our ROI measurements in the frontal WM for NAA (reduced with age), Cho, and tCr concentrations (both showed a tendency of increase with age) are consistent with their observations of age-related reduction of lobar mean NAA concentrations and an increases of lobar mean $\mathrm{tCr}$ and Cho concentrations, ${ }^{3}$ we found that the $\mathrm{tCr}$ concentration was reduced with increasing age in the BSd and putamen, whereas the Cho concentration did not show significant age-related changes in the selected ROIs. These discrepancies may be caused by a regional dependence of age-related metabolic distributions within the lobar structures that was observable only for ROI measurements, as revealed also by a study using both 2D-MR spectroscopy and single-voxel spectroscopy. With 2D MR spectroscopy in the centrum semiovale, Gruber et $\mathrm{al}^{24}$ found a decrease of $3.1 \%$ per decade for NAA and an increase of $3.6 \%$ per decade for Cho, while with single-voxel spectroscopy located in the frontal lobe, they found a positive correlation between $\mathrm{tCr}$ and age, but only a trend of increased Cho with age in the frontal lobe, indicating again the inhomogeneity of metabolite distributions even within a brain lobe.

The tissue transverse relaxation parameters reflect molecularlevel relaxation mechanisms and are sensitive to microstructural variations in tissue, such as changes of the free water content. For example, reduced free water corresponds to shortened T2 in the maturing brain, ${ }^{4,25}$ while pathologic demyelination or neurodegeneration resulting in increased free water content is associated with prolonged T2. ${ }^{26-28}$ In line with these facts, our observations of age-related increase of T2 in cerebellar white matter $(R=0.45)$ may, therefore, reflect an age-related increase of free water within this region, whereas the decrease of T2 at SCC $(R=-0.47)$ may indicate a loss of free water content within that region, though the exact mechanism behind these changes is not yet clear. Siemonsen et $\mathrm{al}^{5}$ found, in a retrospective study of 55 adults (12-90 years of age; mean age, $54 \pm 20$ ), an age-related decrease of $\mathrm{T}^{\prime}$ in the lentiform nucleus $(R=-0.69)$ and no age correlation in the thalamus and parietal white matter, which are consistent with our observations that $\mathrm{T} 2$ ' showed a strong negative linear correlation to age in the putamen $(R=-0.68)$ and no correlation with age in the thalamus and parietal white matter.

Our findings that T2 and T2' values in the motor and occipital cortices were comparable and lowest in the pallidum are consistent with an earlier study on $\mathrm{T} 2$ by Jara et $\mathrm{al}^{29}$ and with regional distribution of nonheme iron in the report by Hallgren and Sourander, ${ }^{30}$ in which the iron content in the motor and occipital cortices was similar while the pallidum had the largest amount of iron. A decrease of $\mathrm{T} 2$ ' in the deep gray matter has been suggested as a marker of increased iron deposition, ${ }^{5,7,8}$ while cortical T2' is considered to provide information about the local concentration of deoxyhemoglobin, which is reduced due to age-related variations of cerebral autoregulation and increased deoxyhemoglobin in elderly subjects. ${ }^{31-33}$ An increase of $\mathrm{T} 2$ ' in the normal-appearing white matter of patients with multiple sclerosis was attributed to reduced metabolism. ${ }^{7}$ Correspondingly, our observations of correlated decreases of T2' and NAA concentrations $(R=0.53)$ in the occipital GM may indicate an association of an age-related increase of deoxyhemoglobin and reductions of neuronal attenuation and/or function in the occipital gray matter. The correlated decreases of T2' with NAA and tCr concentrations ( $R=0.55$ and 0.50 , respectively) in the putamen may indicate associations of age-related increase of iron deposition (decreased $\mathrm{T}^{\prime}$ ) and reductions of neuronal attenuation and/or function (reduced NAA concentrations) and a decrease of energy metabolism (reduced $\mathrm{tCr}$ concentrations) in the deep gray matter, while the observed increase of $\mathrm{T} 2$ ' with age in the white matter of the SCC may reflect reduced metabolism with age, 
consistent with the observations of an age-related reduction of NAA concentrations and T2 in the SCC. Also our observation of a smaller change in $\mathrm{T} 2{ }^{\prime}$ values in the pallidum for ages older than 60 could be an indication of the metabolic function slowing with increased age. The evidence of an age-related increase of iron deposition with reduced neuronal function and energy metabolism in the putamen and age-related reduction of neuronal function, metabolism, and free water in the SCC may make the putamen and corpus callosum more vulnerable to neurodegenerative diseases.

There are several limitations to this study. The differences between the left and right hemispheres and the possible effect of handedness were not considered because of the limited sample size. Due to differences of section thickness (interpolated section thickness of $5.6 \mathrm{~mm}$ for metabolite maps versus $3 \mathrm{~mm}$ for T2 maps), the spatial volumes of ROIs obtained on metabolite maps did not match exactly those on quantitative MR imaging maps. The effect of partial volume contributions from neighboring structures and CSF was also not taken into account. This was, however, minimized by using a small ROI that was visually placed to avoid these contributions. An additional limitation is that subjects older than 70 years were not included due to difficulties in subject recruitment. To overcome these limitations, a study with a larger sample size and more selected brain-specific structures is necessary.

\section{CONCLUSIONS}

This study has demonstrated that age-related metabolic and microstructural changes in the human brain are regionally dependent and more apparent in the cerebrum than in the cerebellum. Correlations between both processes are evident in the gray matter. These results could be used as references for future study on patients.

Disclosures: Andrew A. Maudsley-RELATED: Grant: National Institutes of Health*; UNRELATED: Travel/Accommodations/Meeting Expenses Unrelated to Activities Listed: Helmholtz Association, Comments: International Scholars Award for travel to the Helmholtz research site in Germany. Heinrich Lanfermann-UNRELATED: Expert Testimony: Oberlandesgericht Braunschweig; Grants/Grants Pending: Acandis, ${ }^{*}$ Penumbra*; Payment for Lectures (including service on Speakers Bureaus): Bender Imaging, Radiopharm; Payment for Development of Educational Presentations: Institut für medizinische und pharmazeutische Prüfungsfragen; Travel/Accommodations/Meeting Expenses Unrelated to Activities Listed: German Society of Neuroradiology, German Society of Radiology, Friedrich-Schiller-Universität Jena, Johann Wolfgang GoetheUniversität Frankfurt am Main. Xiao-Qi Ding—RELATED: Grant: German Research Foundation. ${ }^{*}$ Money paid to the institution.

\section{REFERENCES}

1. Haga KK, Khor YP, Farrall A, et al. A systematic review of brain metabolite changes, measured with $1 \mathrm{H}$ magnetic resonance spectroscopy, in healthy aging. Neurobiol Aging 2009;30:353-63 CrossRef Medline

2. Barker PB, Bizzi A, De Stefano N, et al. Clinical MR Spectroscopy: Techniques and Applications. 1st ed. Cambridge: Cambridge University Press, 2009

3. Maudsley AA, Govind V, Arheart KL. Associations of age, gender and body mass with $1 \mathrm{H}$ MR-observed brain metabolites and tissue distributions. NMR Biomed 2012;25:580-93 CrossRef Medline

4. Ding XQ, Kucinski T, Wittkugel O, et al. Normal brain maturation characterized with age-related $\mathrm{T} 2$ relaxation times: an attempt to develop a quantitative imaging measure for clinical use. Invest $R a$ diol 2004;39:740-46 CrossRef Medline

5. Siemonsen S, Finsterbusch J, Matschke J, et al. Age-dependent normal values of T2* and T2' in brain parenchyma. AJNR Am J Neuroradiol 2008;29:950-55 CrossRef Medline

6. Sedlacik J, Boelmans K, Löbel U, et al. Reversible, irreversible and effective transverse relaxation rates in normal aging brain at $3 \mathrm{~T}$. Neuroimage 2014;84:1032-41 CrossRef Medline

7. Holst B, Siemonsen S, Finsterbusch J, et al. T2' imaging indicates decreased tissue metabolism in frontal white matter of MS patients. Mult Scler 2009; 15:701-07 CrossRef Medline

8. Miszkiel KA, Paley MN, Wilkinson ID, et al. The measurement of R2, $\mathrm{R}^{*}$ and $\mathrm{R} 2$ ' in HIV-infected patients using the prime sequence as a measure of brain iron deposition. Magn Reson Imaging 1997;15: 1113-19 CrossRef Medline

9. Steer RA, Clark DA, Beck AT, et al. Common and specific dimensions of self-reported anxiety and depression: the BDI-II versus the BDI-IA. Behav Res Ther 1999;37:183-90 CrossRef Medline

10. Kalbe E, Kessler J, Calabrese P, et al. DemTect: a new, sensitive cognitive screening test to support the diagnosis of mild cognitive impairment and early dementia. Int J Geriatr Psychiatry 2004;19: 136-43 CrossRef Medline

11. Maudsley AA, Domenig C, Sheriff S. Reproducibility of serial wholebrain MR spectroscopic imaging. NMR Biomed 2010;23:251-56 CrossRef Medline

12. Barker PB, Soher BJ, Blackband SJ, et al. Quantitation of proton NMR spectra of the human brain using tissue water as an internal concentration reference. NMR Biomed 1993;6:89-94 CrossRef Medline

13. Abramoff MD, Magalhães PJ, Ram SJ. Image processing with ImageJ. Biophotonics International 2004;11:36-42

14. Baker EH, Basso G, Barker PB, et al. Regional apparent metabolite concentrations in young adult brain measured by (1)H MR spectroscopy at 3 Tesla. J Magn Reson Imaging 2008;27:489-99 CrossRef Medline

15. Pouwels PJ, Brockmann K, Kruse B, et al. Regional age dependence of human brain metabolites from infancy to adulthood as detected by quantitative localized proton MRS. Pediatr Res 1999;46:474-85 CrossRef Medline

16. Kumar R, Delshad S, Woo MA, et al. Age-related regional brain T2-relaxation changes in healthy adults. J Magn Reson Imaging 2012; 35:300-08 CrossRef Medline

17. Li W, Langkammer C, Chou YH, et al. Association between increased magnetic susceptibility of deep gray matter nuclei and decreased motor function in healthy adults. Neuroimage 2015;105: 45-52 CrossRef Medline

18. Ryberg C, Rostrup E, Paulson OB, et al; LADIS study group. Corpus callosum atrophy as a predictor of age-related cognitive and motor impairment: a 3-year follow-up of the LADIS study cohort. J Neurol Sci 2011;307:100-05 CrossRef Medline

19. Brooks JC, Roberts N, Kemp GJ, et al. A proton magnetic resonance spectroscopy study of age-related changes in frontal lobe metabolite concentrations. Cereb Cortex 2001;11:598-605 CrossRef Medline

20. Charles HC, Lazeyras F, Krishnan KR, et al. Proton spectroscopy of human brain: effects of age and sex. Prog Neuropsychopharmacol Biol Psychiatry 1994;18:995-1004 CrossRef Medline

21. Chao LL, Mueller SG, Buckley ST, et al. Evidence of neurodegeneration in brains of older adults who do not yet fulfill MCI criteria. Neurobiol Aging 2010;31:368-77 CrossRef Medline

22. Raininko R, Mattsson P. Metabolite concentrations in supraventricular white matter from teenage to early old age: a short echo time 1H magnetic resonance spectroscopy (MRS) study. Acta Radiol 2010;51:309-15 CrossRef Medline

23. Moreno-Torres A, Pujol J, Soriano-Mas C, et al. Age-related metabolic changes in the upper brainstem tegmentum by MR spectroscopy. Neurobiol Aging 2005;26:1051-59 CrossRef Medline

24. Gruber S, Pinker K, Riederer F, et al. Metabolic changes in the normal ageing brain: consistent findings from short and long

AJNR Am J Neuroradiol 37:447-54 Mar 2016 www.ajnr.org

453 
echo time proton spectroscopy. Eur J Radiol 2008;68:320-27 CrossRef Medline

25. Holland BA, Haas DK, Norman D, et al. MRI of normal brain maturation. AJNR Am J Neuroradiol 1986;7:201-08 Medline

26. Dawe RJ, Bennett DA, Schneider JA, et al. Ex vivo T2 relaxation: associations with age-related neuropathology and cognition. $\mathrm{Neu}$ robiol Aging 2014;35:1549-61 CrossRef Medline

27. Ding XQ, Wittkugel O, Goebell E, et al. Clinical applications of quantitative T2 determination: a complementary MRI tool for routine diagnosis of suspected myelination disorders. Eur J Paediatr Neurol 2008;12:298-308 CrossRef Medline

28. Weissenborn K, Bültmann E, Donnerstag F, et al. Quantitative MRI shows cerebral microstructural damage in hemolytic-uremic syndrome patients with severe neurological symptoms but no changes in conventional MRI. Neuroradiology 2013;55:819-25 CrossRef Medline
29. Jara H, Sakai O, Mankal P, et al. Multispectral quantitative magnetic resonance imaging of brain iron stores: a theoretical perspective. Top Magn Reson Imaging 2006;17:19-30 CrossRef Medline

30. Hallgren B, Sourander P. The effect of age on the non-haemin iron in the human brain. J Neurochem 1958;3:41-51 CrossRef Medline

31. Wagner M, Jurcoane A, Volz S, et al. Age-related changes of cerebral autoregulation: new insights with quantitative $\mathrm{T} 2{ }^{\prime}$-mapping and pulsed arterial spin-labeling MR imaging. AJNR Am J Neuroradiol 2012;33:2081-87 CrossRef Medline

32. Wagner M, Magerkurth J, Volz S, et al. T2' - and PASL-based perfusion mapping at 3 Tesla: influence of oxygen-ventilation on cerebral autoregulation. J Magn Reson Imaging 2012;36:1347-52 CrossRef Medline

33. Seals DR, Jablonski KL, Donato AJ. Aging and vascular endothelial function in humans. Clin Sci (Lond) 2011;120:357-75 CrossRef Medline 